\title{
Decentralized Data-Driven Tuning of Droop Frequency Controllers
}

\author{
Allan Almeida Santos*, Edwin Mora*, Jan Peters*† and Florian Steinke* \\ ${ }^{*}$ Technical University of Darmstadt, Germany \\ Email: \{allan.santos,edwin.mora,florian.steinke\}@eins.tu-darmstadt.de, peters@ias.tu-darmstadt.de \\ ${ }^{\dagger}$ Max Planck Institute for Intelligent Systems, Germany
}

\begin{abstract}
Power oscillation damping is an important task for maintaining electric grid stability and reliability. It is realized via several control mechanisms that are traditionally tuned manually at installation time, often with the help of dynamic simulations of the full grid. With the increasing number of Decentralized Energy Resources (DER) and variable renewable energies, both the grid structure as well as generators' parameters are subject to frequent changes, turning manually tuned controllers suboptimal and existing dynamic models invalid. We therefore develop an automated controller tuning approach for the core mechanism of droop frequency control. It runs decentrally on each generator or in each control area without using an a priori dynamic model of the grid. Instead, we use data-based system identification to estimate a dynamic model of the remaining grid and combine it with local parameters to optimally adapt the local droop value. In simulation experiments, the algorithm is compared against an optimization approach, which has complete knowledge of the full grid configuration, and a heuristic that simulates manual tuning. In the considered setup, our approach reduces frequency oscillations by $15 \%$ compared to simulated manual tuning and comes within $8 \%$ of the all-knowing central approach.
\end{abstract}

Index Terms-Frequency control, Optimal control, Automatic control

\section{INTRODUCTION}

Power oscillations are an intrinsic feature of systems composed of weakly coupled generators [1]. They are excited by changes in the power demand, generator outages, or grid reconfigurations. Oscillations can lead to instabilities or even damage grid equipment in extreme cases, and several control mechanisms are typically employed to dampen them as quickly as possible. Among these are generators' droop frequency control coupled with their inertia, Power System Stabilizers (PSS), and the suitable control of additional hardware such as Static VAR Compensators [1].

The controllers need to be tuned for specific grid configuration, that is characterized by the grid's topology and the parameters of the connected elements. A common approach for controller tuning is to manually adjust parameters based on dynamic grid simulations at installation time or when the grid configuration changes significantly. However, this approach is time-consuming and yields optimal results only for the grid configuration at tuning time. While in the past many power grid configurations were constant over extended time periods, today's many new installations of DERs and renewable generators lead to frequent changes. This makes automated tuning methods a valuable tool for grid adaptation.

Several methods for automated oscillation damping have been developed. Many solutions require new control structures, e.g., [2]-[4]. Such approaches are difficult to implement in systems with a large amount of existing equipment. Another line of work tries to tune existing control structures, e.g., the droop frequency controllers, such that oscillations are optimally damped [5]-[7]. These approaches require a dynamic model of the whole power grid. However, exact dynamic models are difficult to obtain and to maintain in frequently changing environments.

In this work, we therefore propose a decentralized datadriven tuning algorithm for droop frequency control. It is decentralized, meaning that it is executed locally and independently on each generator or in each control area in a continuous manner. It is data-driven, meaning that a dynamic model of the remaining grid as seen from one local agent is estimated using data-based system identification. The algorithm then combines the estimated model with local parameters to obtain optimal local droop gains. In simulation experiments, we show that the proposed approach yields gain values whose performance in oscillation damping in the whole power grid is superior to heuristic manual tuning and not much worse than a central optimization approach that has access to an up-to-date dynamic model of the full grid as well as all possible measurements. Our approach can be implemented on individual devices and can integrate changes of the grid's configuration whenever they occur. It is thus a realistic step to improve oscillation damping in practice.

The remainder of this paper is structured as follows. Section II defines the employed power system model and states our goal. The proposed algorithm is then presented in Section III. In Section IV we evaluate it in comparison with a central optimization baseline and a heuristic manual tuning approach. We conclude the paper in Section V.

\section{Power System Model \& Problem Statement}

We now present the employed power system model including the droop frequency control mechanism. Then, the problem addressed in this work is defined mathematically.

(C)2020 IEEE. Personal use of this material is permitted. Permission from IEEE must be obtained for all other uses, in any current or future media, including reprinting/republishing this material for advertising or promotional purposes, creating new collective works, for resale or redistribution to servers or lists, or reuse of any copyrighted component of this work in other works. 


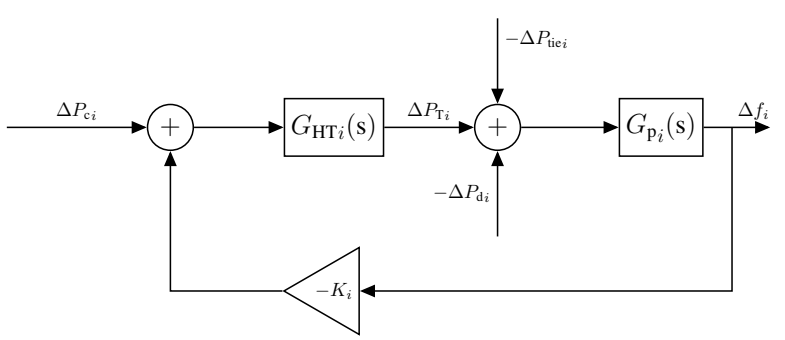

Fig. 1: Block diagram of a control area $i$.

\section{A. Power System Modeling}

We represent the dynamics of the power system via the interaction of $N$ coupled local control areas. Each local area is modeled as a linear dynamical system that relates the local active power demand and the power transfer from other areas to the local power generation and the local system frequency [1]. Since we are interested in oscillations around a working point, we only consider variations of these signals in the following. An overview of the model is given in Fig. 1.

We consider a local control area as a set of power generators strongly coupled due to physical proximity and/or grid's topology. It is assumed that the frequency of all generators in the control area is identical throughout. This allows us to model the control area as a single large power generator with aggregated capacity equal to the sum of each individual generator. For local control area $i$, the relation between the generator's active power set point and its actually generated mechanical power $\Delta P_{\mathrm{T} i}$ is described by the first order transfer function $G_{\mathrm{HT} i}(\mathrm{~s})=\frac{1}{T_{\mathrm{HT} i} \mathrm{~s}+1}$. Hereby, $T_{\mathrm{HT} i}$ is an aggregated time constant for the power generation process. We assume the power set point of the control area scheduled by the secondary or tertiary controller, denoted by $\Delta P_{\mathrm{c} i}$, constant to zero. The transfer function $G_{\mathrm{p} i}(\mathrm{~s})=\frac{K_{\mathrm{p} i}}{\mathrm{~s}}$ represents the behavior of the electrical generator, relating the local power balance to the local system frequency $\Delta f_{i}$. The generator is (de-)accelerated by the balance of the load $\Delta P_{\mathrm{d} i}$, the incoming power from connected control areas $\Delta P_{\text {tie } i}$, and the local power generation $\Delta P_{\mathrm{T} i}$. The gain $K_{\mathrm{p} i}$ is inversely proportional to the inertia $H_{i}$ of the control area. The model considers that each local area is equipped with a droop frequency controller, i.e., a static negative feedback with the gain $K_{i}$, that determines the change in the power production for a given frequency deviation. The gain $K_{i}$ is typically denoted in terms of its inverse value, the droop $d_{i}$. Typical limitations for the droop values are $[1,10] \%$ of the nominal frequency divided by the nominal capacity [8]

Control areas $i$ and $j$ are connected by a tie-line modeled via transfer function $G_{\text {tie }}^{i j}(\mathrm{~s})=\frac{2 \pi L_{i j}}{\mathrm{~s}}$, which relates the inter-area difference of the frequency to the active power flow through the tie-line. $L_{i j}$ is the synchronizing coefficient of areas $i$ and $j$ and is inversely proportional to the line reactance.

\section{B. Problem Statement}

The goal of our algorithm is to damp frequency oscillations in the power grid by tuning the droop gains $d_{i}$ locally in each area.
A local measure of frequency oscillations is the timevariance of the frequency deviations,

$$
\phi_{i}=\frac{1}{T-1} \sum_{t=1}^{T}\left(\Delta f_{i, t}-\mu_{i}\right)^{2},
$$

with $\Delta f_{i, t}$ being the frequency of control area $i$ sampled at time step $t, T$ the number of collected samples, and $\mu_{i}$ being the average of the frequency of control area $i$ over the $T$ samples. The performance of a set of droop gains for all areas is then evaluated via the average variance in all areas, i.e.,

$$
\phi=\frac{1}{N} \sum_{i=1}^{N} \phi_{i} .
$$

Both measures are dependent on the excitation signal, that is, the load signal applied to the system, and are only comparable for identical setups. We use square wave excitations in the following. To remove the stationary control error and amplify small frequency oscillations, we normalize the frequency samples before computing the time-variance. Let $\mathcal{T}=\{0, \epsilon, 2 \epsilon, \ldots,(T-1) \epsilon\}$ be the set of time steps when the frequency deviation signal was sampled, with $\epsilon$ being the sampling period. We define $\mathcal{S} \subset \mathcal{T}$ as the set of time steps when a change in the input value occurred during the frequency sampling. The normalized frequency is then given by

$$
\Delta \hat{f}_{i, t}=\frac{\Delta f_{i, t}-\frac{1}{|\sigma(t)|} \sum_{\tau \in \sigma(t)} \Delta f_{i, \tau}}{\left|\max _{\tau \in \sigma(t)} \Delta f_{i, \tau}-\min _{\tau \in \sigma(t)} \Delta f_{i, \tau}\right|},
$$

where $\sigma(t)=\{\tau \in \mathcal{T} \mid \tau \geq \max \{m \in \mathcal{S} \mid m \leq t\}, \quad \tau<$ $\min \{m \in \mathcal{S} \mid m>t\}\}$ and $|\sigma(t)|$ denotes the cardinality of set $\sigma(t)$.

\section{Decentralized Data-Driven Tuning}

The algorithm developed in the following is composed of two parts. First, a data-based system identification step is performed, where each area estimates a model of the remaining, non-local grid. Thereafter, the droop controller is optimized locally given the estimated model and the local parameters of the control area.

Data-Based System Identification: We first model the remaining grid as seen from local control area $i$ as a single transfer function $G_{\mathrm{r} i}(\mathrm{~s})$ with only local inputs and outputs. Fig. 2 shows $G_{\mathrm{r} i}(\mathrm{~s})$ interconnected with control area $i$. The input of this transfer function is the local frequency deviation $\Delta f_{i}$ and its output $\Delta P_{\text {tie } i}$ is the sum of the incoming power of all tie-lines connected to control area $i$.

$G_{\mathrm{r} i}(\mathrm{~s})$ is estimated using the Canonical Variate Analysis algorithm (CVA) [9], which is within the class of subspace methods for system identification [10]. It was verified experimentally that this method provides a more accurate model of the remaining grid compared to other subspace methods, such as MOESP [11] and SSARX [12].

For performing the estimation, we excite the local load $\Delta P_{\mathrm{d} i}$ during a time window of $100 \mathrm{~s}$ with two consecutive step signals with uniformly distributed random amplitude between 


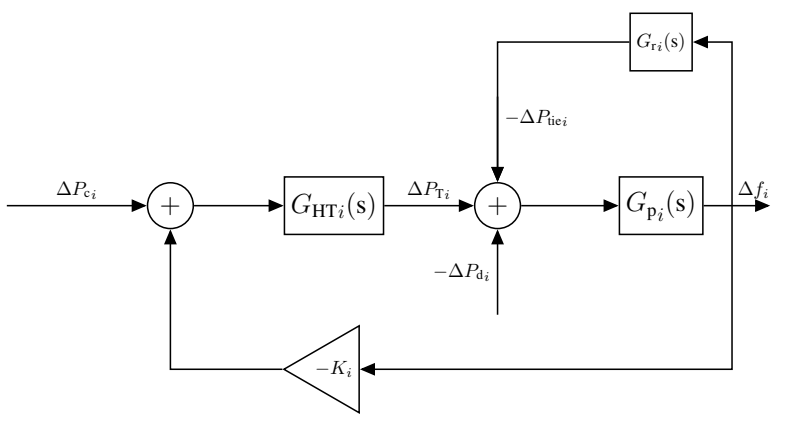

Fig. 2: Block diagram of a control area $i$ with the remaining of the power grid modeled as $G_{\mathrm{r} i}(\mathrm{~s})$.

$[-1,1]$ p.u. of power. The time window length was found experimentally and was the smallest value in order to produce satisfactory results for the estimation of $G_{\mathrm{r} i}(\mathrm{~s})$. During this experiment we collect $10^{3}$ samples of the input/output data pair $\left(\Delta f_{i}, \Delta P_{\text {tie } i}\right)$ to be used in the estimation algorithm. In our experiments, we collected the data from a simulated model of the whole power grid. In a real-world implementation, however, this data shall be collected from the physical power grid by using suitable measurement equipment.

Observe that the order of the system to be estimated is a hyperparameter of the CVA algorithm. Therefore, as the order of the remaining grid seen by the local area is unknown, we perform hyperparameter optimization using grid search. The employed performance metric is an online evaluation of the grid that consists of calculating $\phi_{i}$ over $500 \mathrm{~s}$ with $10^{4}$ samples of the frequency deviation of the local control area. As for the system identification, we collected the data from a simulated model of the power grid. During the evaluation, the loads of all control areas are excited with square signals with independent and identically distributed (i.i.d.) random amplitudes uniformly distributed between $[-1,1]$ p.u. with period of $10 \mathrm{~s}$. In our experiments, we limited the search to order 10 , both to maintain acceptable computation times as well as to limit overfitting effects. The aforementioned online evaluation is performed after the optimization step described in the following.

Droop Optimization: With an estimation of $G_{\mathrm{r} i}(\mathrm{~s})$ from the previous step, we utilize the logarithmic barrier method (LBM) [13] with numerically computed gradients to find the optimal droop value $d_{i}$ of control area $i$ given the physical constraints stated in Section II, i.e., we perform the following minimization

$$
\min _{d_{i}} \phi_{i}\left(d_{i}\right)-(1 / \rho)\left[\log \left(-g\left(d_{i}\right)\right)+\log \left(h\left(d_{i}\right)\right)\right],
$$

with $g\left(d_{i}\right)=d_{i}-10 \%$ and $h\left(d_{i}\right)=d_{i}-1 \% . \rho \in \mathbb{R}^{+}$defines the quality of the approximation of the barrier function. $\phi_{i}$ is computed by simulating the model of the local control area connected to the estimated remaining of the power grid as in Fig. 2. The model is perturbed by exciting $\Delta P_{\mathrm{d} i}$ for $500 \mathrm{~s}$ with a square signal with random amplitude uniformly distributed between $[-1,1]$ p.u. with period of $10 \mathrm{~s}$.
The pseudocode for the decentralized data-driven droop tuning is presented in Algorithm 1. $d_{i}^{*}$ represents the optimal value of $d_{i}$ and is initially set to the mean value of the feasible droop set. $\kappa$ is the order of the estimated system, and $\operatorname{SYS}\left(d_{i}\right)$ refers to the power grid model as in Fig. 2 with droop $d_{i}$. eval represents the online evaluation of the grid with the droop of control area $i$ set to $d_{i}$, which is obtained from the LBM.

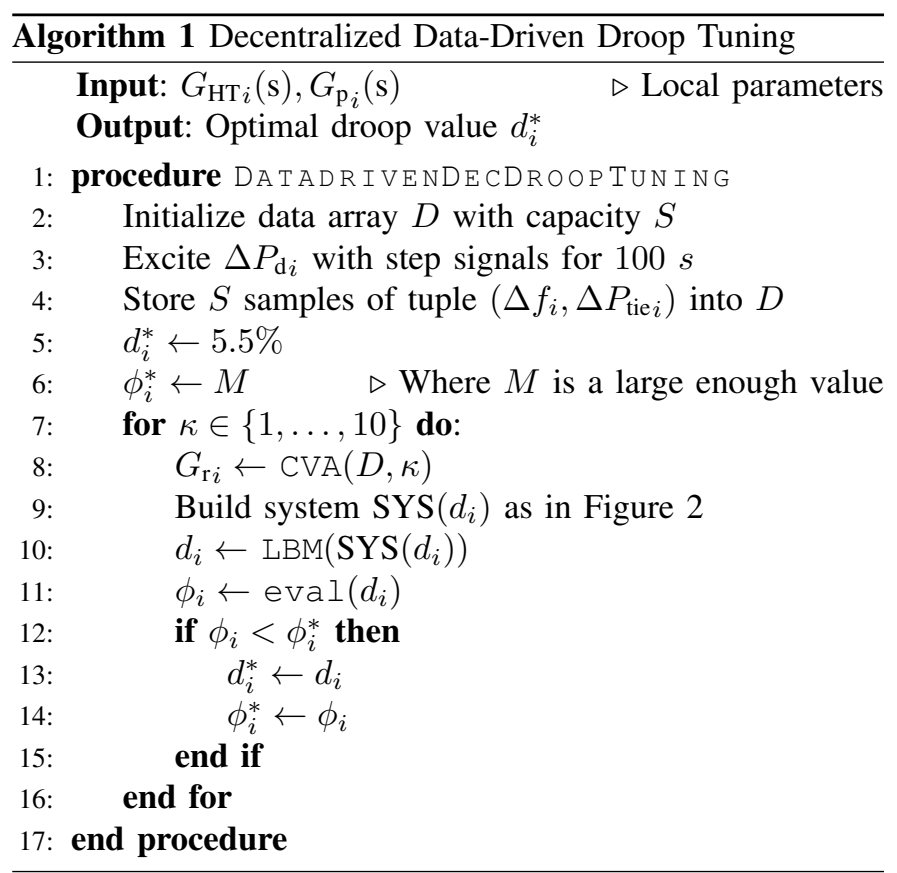

\section{EXPERIMENTS}

We now compare our decentralized data-driven tuning algorithm with a baseline that has complete knowledge of the grid parameters and measurements, and with a heuristic that simulates manual tuning. The algorithms are evaluated for two scenarios in which we suddenly modify the grid topology by connecting a new control area into the grid. Thereafter, we describe the simulation setup and then comment on the results.

\section{A. Algorithms' Implementation}

Decentralized Data-Driven Tuning: Provided that the algorithm is implemented in all control areas of the power grid, it is assumed that all of its instances perform the optimization at the same time and that there are no interferences between them during the system identification step. These assumptions were necessary for simplification of the simulation setup. In a real-application, however, synchronization of the algorithm's instances would not be required and interferences would rarely occur and/or could be easily avoided, e.g., by applying random delays.

Baseline: A centralized version of our algorithm is used as a baseline. It performs the LBM again with numerically computed gradients to optimize the set of droops $\mathbf{d}=$ $\left\{d_{1}, \ldots, d_{N}\right\}$ provided the up-to-date full grid model and the frequency deviation measurements from the $N$ control 

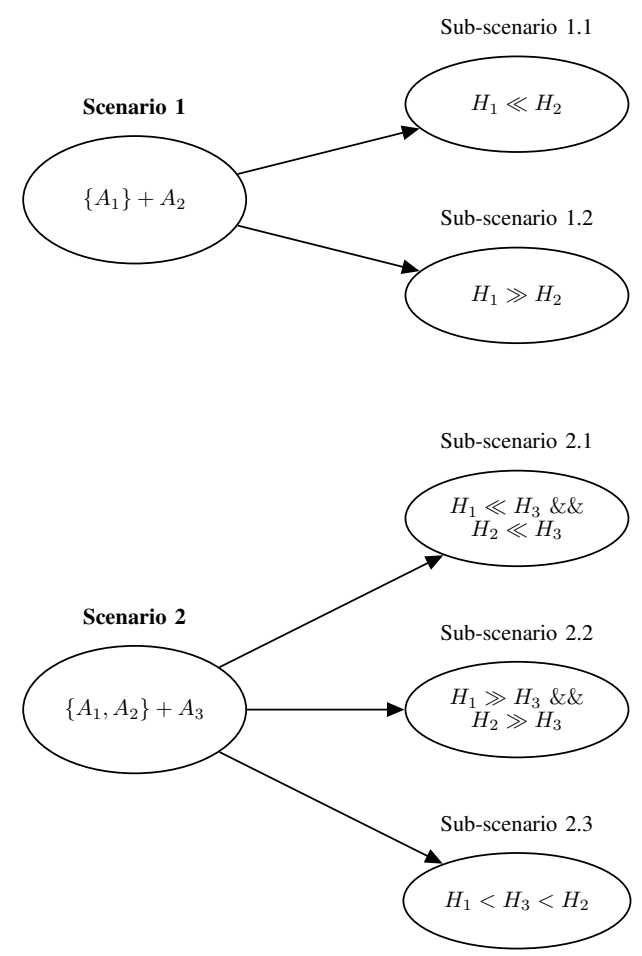

Fig. 3: Structure of the examined test scenarios.

areas. The LBM optimizes for the average performance of the interconnected control areas $\phi$.

Manual Tuning: We simulate the manual tuning of the control areas by applying the following heuristic that worked well in many simulation experiments. For any grid setting, the droop value of the control area with largest inertia is set to $8 \%$. For all other interconnected control areas the droop value is set to $2 \%$. However, supposing that manual adaption will not happen immediately after a change in the grid configuration, areas that are newly connected within one simulation have their droop values set to the default value of $5.5 \%$ independently of their relative inertia.

\section{B. Study Scenarios Definition}

The designed study scenarios simulate the connection of a new control area into the power grid. The withdrawal of a control area from the grid is an analogous scenario and, thus is not simulated. Within each scenario we utilize different values for the inertia of the control areas, as we aim to cover most of the situations that could happen in the operation of a real power grid. Fig. 3 summarizes the study scenarios and their sub-scenarios.

We define Scenario 1 as connecting a control area $A_{2}$ into a single-area power grid containing $A_{1}$. We then divide it into two sub-scenarios. Sub-scenario 1.1 has $A_{1}$ with total inertia $H_{1}=8 \mathrm{~s}$ and $A_{2}$ with $H_{2}=4 \mathrm{~s}$. In Sub-scenario 1.2 , the inertia values are swapped. Scenario 2 is defined as connecting a control area $A_{3}$ into a power grid with two interconnected control areas $A_{1}$ and $A_{2}$ with inertias $4 s$ and $8 s$, respectively. It is assumed that $A_{3}$ is connected at the same time to both

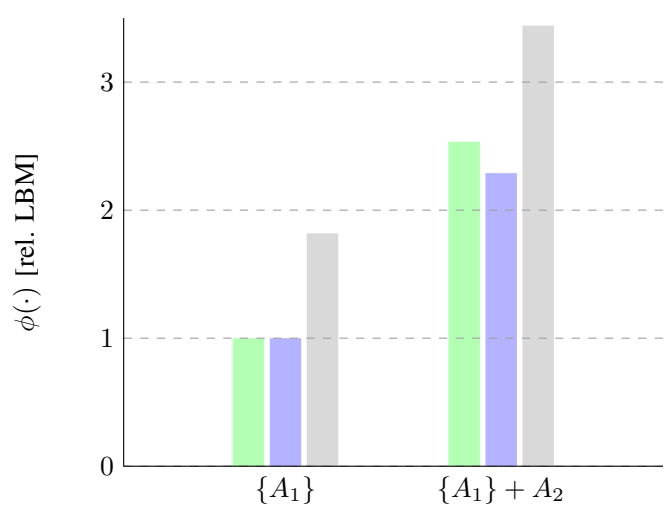

(a)

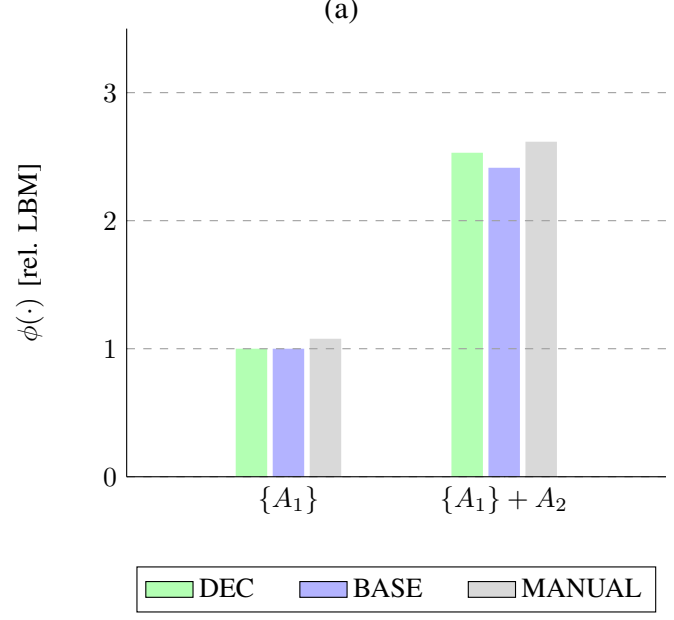

(b)

Fig. 4: Performance metric $\phi$ for Scenario 1 (a) with parameters of Sub-scenario 1.1 and (b) with parameters of Subscenario 1.2.

$A_{1}$ and $A_{2}$, thus forming a ring-topology. From this case, we can define three sub-scenarios. In Sub-scenario 2.1 the inertia constant of $A_{3}$ is set to $H_{3}=16 \mathrm{~s}$, which is much greater than the total inertia of the power grid. In Sub-scenario 2.2, $A_{3}$ has inertia set to $2 \mathrm{~s}$, much smaller than both $H_{1}$ and $H_{2}$. At last, we define Sub-scenario 2.3 as $A_{3}$ having intermediate inertia, with $6 \mathrm{~s}$. In the aforementioned scenarios, $T_{\mathrm{HT} i}$ is set to $10 \%$ of $H_{i}$ for all control areas, and all tie-line gains $L_{i j}$ are set to $2 \pi p . u$. of power.

\section{Evaluation Criterion}

In both study scenarios, the algorithms' performance is evaluated by calculating the average frequency variance $\phi$ in the power grid. To simulate disturbance in the grid, the load of the control areas are excited by square signals with i.i.d. random amplitudes uniformly distributed between $[-1,1] p . u$. with period of $10 \mathrm{~s}$. The power grid with the configuration previous to the connection of the new control area is then evaluated by computing $\phi$ for a time window of $500 \mathrm{~s}$ using $10^{4}$ frequency deviation samples. Thereafter, the algorithms optimize the set of droops for the grid configuration with the additional control area. The evaluation is performed for a 
TABLE I: Performance metric $\phi$ for Scenario 2

\begin{tabular}{|c|c|c|c|c|c|c|}
\hline & \multicolumn{2}{|c|}{ DEC } & \multicolumn{2}{|c|}{ BASE } & \multicolumn{2}{|c|}{ MANUAL } \\
\hline & $\left\{A_{1}+A_{2}\right\}$ & $\left\{A_{1}+A_{2}\right\}+A_{3}$ & $\left\{A_{1}+A_{2}\right\}$ & $\left\{A_{1}+A_{2}\right\}+A_{3}$ & $\left\{A_{1}+A_{2}\right\}$ & $\left\{A_{1}+A_{2}\right\}+A_{3}$ \\
\hline Sub-scen. 2.1 & 1.05 & 3.33 & 1 & 3.11 & 1.09 & 5.83 \\
\hline Sub-scen. 2.2 & 1.05 & 3.45 & 1 & 3.13 & 1.09 & 3.59 \\
\hline Sub-scen. 2.3 & 1.05 & 5.98 & 1 & 4.66 & 1.09 & 6.03 \\
\hline
\end{tabular}

subsequent period of $500 s$ using also $10^{4}$ frequency deviation samples.

Observe that this evaluation is different from the one performed for the grid search in the system identification step. The latter utilizes only the local frequency deviation to determine the grid performance, as only local knowledge is assumed, while the former applies the frequency deviation of all control areas to determine the overall performance of the algorithms.

\section{Experimental Results}

Fig. 4 shows the performance comparison for the two sub-scenarios of Scenario 1 and Table I for the three subscenarios of Scenario 2, with DEC standing for the developed decentralized data-driven algorithm, BASE for the centralized baseline, and MANUAL for the manual tuning approach. The values are shown relative to the baseline performance of the first time segment, before a new area is connected.

The developed method performed better than the manual approach in all sub-scenarios with average improvement of approximately $14.8 \%$. It performed on average approximately $7.9 \%$ worse than the baseline, which was expected as the developed algorithm uses an estimation of the whole grid, and has therefore an additional error source compared to the baseline.

This demonstrates that the developed algorithm can be a good solution for tuning droop frequency controllers in a power grid with frequent changes in its configuration. It can outperform manual tuning and is not much worse than a centralized tuning method. Its advantage compared to the latter is that it does not rely on the up-to-date grid model nor on communication with a central node, as each control area is able to perform the droop optimization with only local data.

\section{CONCLUSION}

The developed algorithm for tuning the droop frequency control enables frequency oscillation damping using a decentralized approach independent of the current grid configuration by using data-based system identification. It performs $15 \%$ better than manual tuning and has comparable performance with a baseline with complete knowledge of the power grid. The usage of this algorithm helps improve the stability and reliability of the future power grid, in which continuous reconfigurations with new decentral generators or active prosumers will become an inherent feature.

Future work will focus on testing additional methods for system identification and on applying the algorithm to further oscillation damping mechanisms and to larger grids with more control areas.

\section{REFERENCES}

[1] P. Kundur, N. J. Balu, and M. G. Lauby, Power system stability and control. McGraw-hill New York, 1994, vol. 7.

[2] R. Preece, J. V. Milanović, A. M. Almutairi, and O. Marjanovic, "Damping of inter-area oscillations in mixed ac/dc networks using wams based supplementary controller," IEEE Transactions on Power Systems, vol. 28, no. 2, pp. 1160-1169, 2012.

[3] A. Fuchs, M. Imhof, T. Demiray, and M. Morari, "Stabilization of large power systems using vsc-hvdc and model predictive control," IEEE Transactions on Power Delivery, vol. 29, no. 1, pp. 480-488, 2013.

[4] R. A. Biroon, P. Pisu, and D. Schoenwald, "Inter-area oscillation damping in large-scale power systems using decentralized control," in ASME 2018 Dynamic Systems and Control Conference. American Society of Mechanical Engineers Digital Collection, 2018.

[5] A. L. Do Bomfim, G. N. Taranto, and D. M. Falcao, "Simultaneous tuning of power system damping controllers using genetic algorithms," IEEE Transactions on Power Systems, vol. 15, no. 1, pp. 163-169, 2000.

[6] A. Mešanović, D. Unseld, U. Münz, C. Ebenbauer, and R. Findeisen, "Parameter tuning and optimal design of decentralized structured controllers for power oscillation damping in electrical networks," in 2018 Annual American Control Conference (ACC). IEEE, 2018, pp. 38283833.

[7] T. Okada, T. Watanabe, and K. Yasuda, "Parameter tuning of fixed structure controller for power system stability enhancement," in IEEE/PES Transmission and Distribution Conference and Exhibition, vol. 1, Oct 2002, pp. 162-167 vol.1.

[8] "Ieee recommended practice for functional and performance characteristics of control systems for steam turbine-generator units," IEEE Std 122-1991, pp. 1-28, April 1992.

[9] W. E. Larimore, "Canonical variate analysis in identification, filtering, and adaptive control," in 29th IEEE Conference on Decision and control. IEEE, 1990, pp. 596-604.

[10] T. Katayama, Subspace methods for system identification. Springer Science \& Business Media, 2006.

[11] M. VERHAEGEN and P. DEWILDE, "Subspace model identification part 1. the output-error state-space model identification class of algorithms," International Journal of Control, vol. 56, no. 5, pp. 1187-1210, 1992. [Online]. Available: https://doi.org/10.1080/00207179208934363

[12] M. Jansson, "Subspace identification and arx modeling," in Proceedings of the 13th IFAC SYSID Symposium, 2003, pp. 1625-1630.

[13] S. Boyd and L. Vandenberghe, Convex optimization. Cambridge university press, 2004. 